\title{
Nuclear clusters in dwarf irregular galaxies and their connection to massive galactic clusters
}

\author{
Iskren Y. Georgiev, ${ }^{1,2}$ Michael Hilker, ${ }^{3}$ Thomas H. Puzia, ${ }^{4}$ \\ Paul Goudfrooij ${ }^{2}$ and Holger Baumgardt ${ }^{1}$ \\ ${ }^{1}$ Argelander-Institut für Astronomie, Universität Bonn, Auf dem Hügel 71, 53121 Bonn, \\ Germany \\ ${ }^{2}$ Space Telescope Science Institute, Baltimore, MD, USA \\ ${ }^{3}$ ESO, Garching, Germany \\ ${ }^{4}$ Dominion Astrophysical Observatory, Herzberg Institute of Astrophysics, \\ National Research Council of Canada, Victoria, BC, Canada
}

\begin{abstract}
We compare nuclear globular clusters (nGCs) in dwarf galaxies and Galactic GCs with extended (hot) horizontal branches (EHB-GCs) to test the suggested external origin of the latter and the conditions under which GC self-enrichment can operate. We show that the present-day escape velocity $\left(v_{\text {esc }}\right)$ of stellar ejecta to reach the cluster tidal radius compares with those of EHB-GCs. For EHB-GCs, we find a correlation between the present-day $v_{\text {esc }}$ and their metallicity as well as $(V-I)$ colour. The similar $v_{\text {esc }}$ and $(V-I)$ distribution of nGCs and EHBGCs implies that nGCs could also have complex stellar populations. The $v_{\mathrm{esc}}-[\mathrm{Fe} / \mathrm{H}]$ relation could reflect the known relation of increasing stellar-wind velocity with metallicity, which in turn explains why more metal-poor clusters typically show more peculiarities in their stellar population than more metal-rich clusters of the same mass.
\end{abstract}

Keywords. galaxies: star clusters, galaxies: dwarf, galaxies: nuclei

The full poster (in pdf format) is available at http://www. astro.iag.usp.br/ iaus266/Posters/pGeorgiev.pdf. 